\title{
Assessing the Knowledge of Nurses Regarding Hypertension Management Who are Working in National Institute of Cardiovascular Diseases and Hospital in Bangladesh Chowdhury Urmi Rahman ${ }^{1}$, Fatema Tuj Johara ${ }^{2}$, s M Ferdous Hossain ${ }^{3}$ \\ ${ }^{1}$ College of Nursing, University of Dhaka, Dhaka-1000, Bangladesh chow.81may@gmail.com \\ ${ }^{2}$ Marketing Research, Pacific Bangladesh Telecom Limited, 14, C/A, Mohakhali, Dhaka, Bangladesh fjohara@isrt.ac.bd \\ ${ }^{3}$ Enterprise Business Intelligence, Robi Axiata Limited, Gulshan-1, Dhaka, Bangladesh smhossain@isrt.ac.bd
}

\begin{abstract}
Hypertension is a chronic disease, which progresses silently and leads to many complications affecting major body organs. Hypertensive patient's awareness may be developed on the various aspects regarding disease and self-care management through the health care providers. Nurses knowledge regarding hypertension plays an important role in the ability to the management of hypertension Patients [Kaur et al., 2007]. This descriptive type of cross-sectional study was conducted to assess the Nurses knowledge regarding hypertension in National Institute of Cardiovascular Diseases, (NICVD), Sher-E-Bangla Nagar, Dhaka, Bangladesh. Sample size was selected 60 nurses through purposive sampling technique. Data were collected by using the self-administer questionnaire through maintaining face to face interview of the respondents. Data analysis procedure was done by using statistical approaches. Analyzing demographic information, it has found, most of the nurses are from older age group with majority of them belong to secondary level education, significant proportion of them have done diploma in nursing. In terms of awareness, largest number of respondents has very good knowledge on concept of hypertension. They have also fairly known about management and prevention of hypertension. However, they have limitation in knowing causes and symptom of hypertension. In addition, they have quite poor knowledge how to control hypertension. From this study, it is concluded that, hospital management require instigating different training opportunities and learning session so that nurses would have improve their knowledge.
\end{abstract}

Keywords: Metabolic diseases, Judgmental sampling, Questionnaire, Chronic disease, Hypertension, Cardiovascular disease

\section{INTRODUCTION}

Hypertension is the third leading risk factor contributing to death Worldwide. It is one of the significant public health problems in both developing and developed countries. Hypertension is controllable non-communicable diseases. Uncontrolled hypertension has serious consequences including stroke, coronary heart disease, heart failure, and kidney disease [Addo et al., 2007]. In the year 2000, there were 972 million people living with hypertension globally, and this number will escalate to more than 1.56 billion by the year 2025 [Who, 2012].

Prevalence rate of hypertension in Bangladesh was 30.64\% [Rahim-Williams et al., 2012]. Hypertension usually does not cause symptoms initially, but sustained hypertension over time is a major risk factor for hypertensive heart disease, coronary artery disease, stroke, aortic aneurysm, peripheral artery disease, and chronic kidney disease.

To maintain complicity and instant management of situation, it is obvious to have knowledge regarding hypertension by nurses. That is nurses knowledge regarding hypertension management plays an important role for the hypertensive patients through developing their ability to keep control in stable as well as rehabilitation in normal life. There are a number of studies conducted to assess public's knowledge of hypertension management. However, hypertension management and awareness by nurses are still limited in existing literature. Concerning the limitation of study an attempt has taken to assess nurses' knowledge regarding hypertension.

A number of existing literatures has taken under consideration in order to identify assessment criteria among nurses. Several studies revealed that lifestyle, such as physical activity and nutrition, plays an important role in controlling hypertension and preventing its long-term complications [Ard and Svetkey, 2005]. Inadequate nurses' knowledge and awareness about blood pressure (BP) are also potential causes for non-adherence to taking anti-hypertensive drugs, and consequently, high rates of uncontrolled BP.

An assessment of knowledge of high blood pressure ought to be a starting point for educational strategies that aim to deepen understanding of state of health [Viera et al., 2008]. Hence assessing the knowledge, perception, attitudes and lifestyle practices of hypertensive patients is vitally important in achieving hypertension control goals at the population level and also for meeting quality standards in healthcare delivery. And such delivery can primarily be provided by nurses. 
Hypertension can be maintained through restriction of salt, avoid fatty food, reduce heavy alcohol consumption, add potassium and oily fish, stop smoking, regular exercise, weight reduction, relaxation like praying, meditation [Shakhawat Hossain and Martensson, 2008].

Reviewing the above literatures, a number of variables have been considered to make assessment of nurses' knowledge regarding hypertension. Therefore a cross-sectional study has been conducted in aiming to assess the level of nurses knowledge regarding hypertension management who are involved in caring for admitted hypertensive patients at this tertiary level hospital in Bangladesh.

The specific aims of the study were to assess the level of nurses' knowledge regarding-

- Concept of hypertension.

- Management of hypertension.

- Preventive measures of hypertension.

- Complications of hypertension.

Besides, to find out nurses' socio-demographic characteristics.

\section{METHODS}

The study was conducted in male medical and female medical ward in national institute of cardiovascular hospital (NICVD), Sher-E-Bangla Nagar, Dhaka. It is a 414 bedded tertiary level of specialized hospital where all types of hypertensive patients admitted for proper treatment and better facilities.

A sample of size 60 nurses was taken under consideration. Samples were selected by judgmental sampling design. A self administered questionnaire was prepared to select required information for the study. The questionnaire was consisted of two sections; first one covered the demographic information and second section contained 15 (fifteen) questions related to knowledge on hypertension management. Each questions contained 4 (four) options. The questions were multiple responses in nature.

\section{RESULTS AND DISCUSSION}

This section represents results and discussion based on acquired information. First of all, demographic information was represented with frequencies and percentages.

Table 1: Respondents Demographic Information

\begin{tabular}{llcl}
\hline \hline Demographics & Categories & Frequency & Percentage \\
\hline Age & & & \\
\hline & 25 to 35 & 12 & 20.0 \\
& 36 to 45 & 24 & 40.0 \\
& 46 to 55 & & 40.0 \\
& & 59 & 98.3 \\
Gender & Female & 1 & 1.70
\end{tabular}

\begin{tabular}{llcl}
\hline Religion & Islam & 38 & 63.3 \\
& Hindu & 17 & 28.3 \\
& Christian & 4 & 6.70 \\
& Buddhism & 1 & 1.70 \\
& & & \\
\hline Marital Status & Married & 59 & 98.3 \\
& Unmarried & 1 & 1.70 \\
& & & \\
\hline Education Level & Secondary & 48 & 80.0 \\
& Higher secondary & 7 & 11.7
\end{tabular}


Masters $\quad 2 \quad 3.30$

\begin{tabular}{llll}
\hline Professional & Diploma in Nursing & 43 & 71.7 \\
& BSc in Nursing & 10 & 16.7 \\
& MPH & 7 & 11.7 \\
\hline
\end{tabular}

The table 1 shows demographic information of respondents. It has observed that majority of respondents (98\%) are female. Middle age and elder age group people have significant participation in nursing profession. Among respondents significant proportions are from Muslim (63\%), followed by Hindu religion with 23\%. Largest percentage of respondents is married (98\%). Exactly $80 \%$ respondents are just secondary level passed and $8 \%$ respondents completed their higher education in terms of Bachelor and masters. More than $70 \%$ respondents have professional degree diploma in nursing, however very few respondents $11 \%$ have higher degree $\mathrm{MPH}$.

Knowledge based information were represented with frequency distribution of correct and incorrect information of multiple responses.

Table 2: Respondents knowledge regarding concept of hypertension

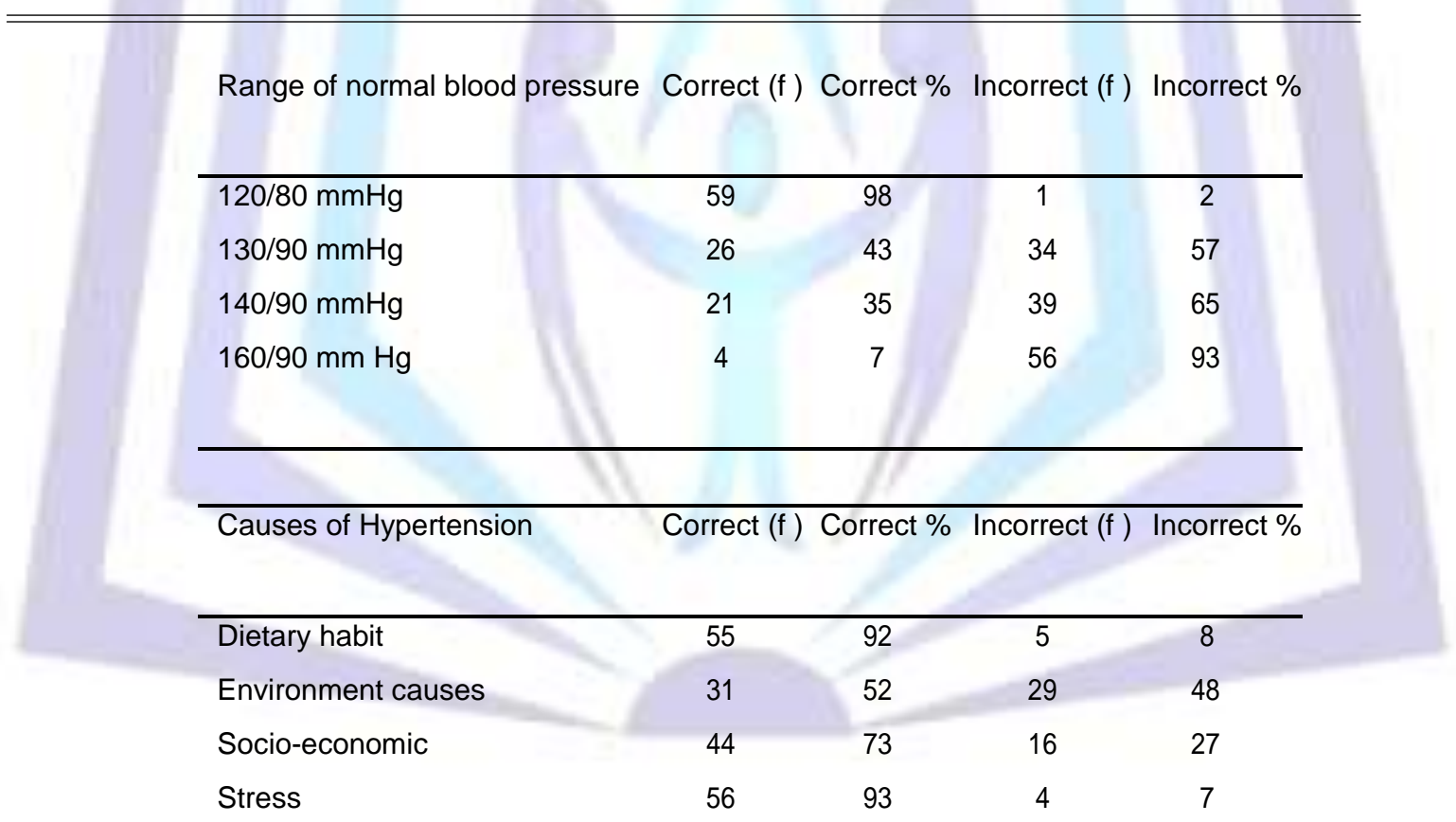

Drugs develop hypertension $\quad$ Correct (f ) Correct \% Incorrect (f) Incorrect \%

\begin{tabular}{lcccc}
\hline Analgesia & 12 & 20 & 48 & 80 \\
Antibiotic & 4 & 7 & 56 & 93 \\
Parkinsonism & 20 & 33 & 40 & 67 \\
Steroids & 59 & 98 & 1 & 2 \\
& & & & \\
\hline
\end{tabular}




\begin{tabular}{lcccc}
\hline Factors & Correct (f ) & Correct \% & Incorrect (f) & Incorrect \% \\
& & & & \\
\hline Age & 57 & 95 & 3 & 5 \\
Genetic factor & 60 & 100 & - & 0 \\
Obesity & 55 & 92 & 5 & 8 \\
Sex & 44 & 73 & 16 & 27 \\
& & & & \\
\hline
\end{tabular}

\begin{tabular}{llll}
\hline Mostly venerable group $\quad$ Correct (f ) & Correct \% & Incorrect (f ) & Incorrect \%
\end{tabular}

\begin{tabular}{lcccc}
\hline Metabolic diseases & 46 & 77 & 14 & 23 \\
Obesity & 58 & 97 & 2 & 3 \\
Old age & 53 & 88 & 7 & 12 \\
Stress & 58 & 97 & 2 & 3 \\
& & & & \\
\hline & & & & \\
\hline Symptoms of hypertension & Correct (f) & Correct \% & Incorrect (f) & Incorrect \% \\
& & & & 10 \\
\hline Abdominal pain & 2 & 3 & 58 & 40 \\
Aching pain in the back of neck & 54 & 90 & 6 & 0 \\
Dizziness & 36 & 60 & 24 & - \\
Headache & 60 & 100 & & \\
\hline
\end{tabular}

From the table 2 it can be seen that, in some areas of knowledge based questions, nurses were found more knowledgeable like on the area of conception part of hypertension, the majority $(93 \%)$ of the respondents could define hypertension correctly. In the area of the normal range of hypertension and responsible drugs for developing hypertension, $98 \%$ respondents answered correctly. Regarding the causes of hypertension, respondents emphasized on dietary habit and stress (above 95\%). On the other hand, nearly 50\% nurses were incorrect about environmental cause and socioeconomic factor. Moreover $80 \%$ and more respondents had misled knowledge on development of hypertension by drugs. Respondents were spontaneous concerning the genetic factors as more than $80 \%$ responses were correct. Moreover more than $85 \%$ responses were correct for knowledge regarding most vulnerable group suffer from hypertension. This might be because of wide spread sharing of knowledge through socio-economic relationship. Respondents have very selective knowledge when it comes to know the symptoms of hypertension as they only aware of common symptoms, aching pain from the back $(90 \%)$, and headache $(100 \%)$. This knowledge gap focused on that point that the nurses had no special training on this area and there is a long gap between their accomplishments of academic courses and current practice.

Table 3: Respondents knowledge regarding management of hypertension

Immediately management

Correct (f) Correct \% Incorrect (f) Incorrect \%

\begin{tabular}{lcccc}
\hline Absolute bed rest & 60 & 100 & 0 & 0 \\
Administer oxygen inhalation if feel suffocation & 40 & 67 & 20 & 33
\end{tabular}


Loosing tight clothing

Provide suggested relaxant
56

54
93

90
4

6
7

10

Type of food $\quad$ Correct (f) Correct \% Incorrect (f) Incorrect \%

\begin{tabular}{lcccc}
\hline Less carbohydrate & 42 & 70 & 18 & 30 \\
Low fatty diet & 57 & 95 & 3 & 5 \\
Vegetables & 51 & 85 & 9 & 15 \\
All of the above & 45 & 75 & 15 & 25
\end{tabular}

\begin{tabular}{llll}
\hline Instruction to discharge & Correct $(f)$ Correct \% & Incorrect $(f)$ Incorrect \%
\end{tabular}

\begin{tabular}{lcccc}
\hline Light exercise & 59 & 98 & 1 & 2 \\
Restricted fatty food & 60 & 100 & 0 & 0 \\
Routinely rest and sleep & 55 & 92 & 5 & 8 \\
Smoking strictly prohibited & 59 & 100 & 0 & 0 \\
& & & \\
\hline
\end{tabular}

The table 3 shows alongside in case of immediate management of hypertension, more than $80 \%$ respondents answered correctly. Moreover, they had sound knowledge on food type (80\%) and immediate hospitalization (83\%) and instruction for discharged patients (95\%) for hypertensive patients. This good knowledge might be due to the fact that the nurses working in NICVD as specialized hospital and getting scope to take history of patients during providing care to the patients including this aspect.

Table 4: Respondents knowledge regarding prevention of hypertension

\begin{tabular}{lcccc}
\hline \hline & & & \\
& Prevention of hypertension & Correct $(f)$ & Correct \% Incorrect ( $f$ ) & Incorrect \% \\
\cline { 3 - 3 } dietary habit & 60 & 100 & 0 & 0 \\
Reduce tension & 60 & 100 & 0 & 0 \\
Regularly exercise & 54 & 90 & 6 & 10 \\
Weight reduction & 60 & 100 & 0 & 0
\end{tabular}

Controlling hypertension $\quad$ Correct (f ) Correct \%Incorrect (f ) Incorrect \%

\begin{tabular}{lcccc}
\hline Alcohol & 60 & 100 & 0 & 0 \\
Extras salt & 60 & 100 & 0 & 0 \\
Fast food & 54 & 90 & 6 & 10 \\
Smoking & 59 & 98 & 1 & 2 \\
& & & & \\
\hline
\end{tabular}


From table 4, in terms of preventing and controlling hypertension nurses have comprehensive knowledge as they provided nearly $100 \%$ correct answer respectively.

Table 5: Respondents knowledge regarding complications of hypertension

\begin{tabular}{lcccc}
\hline \hline Immediate Complications & Correct (f) & Correct \% & Incorrect (f) & Incorrect \% \\
& & & & \\
\hline Faint & 36 & 60 & 24 & 40 \\
Heart Attack & 54 & 90 & 6 & 10 \\
Renal failure & 34 & 57 & 26 & 43
\end{tabular}

\begin{tabular}{llllc}
\hline \multicolumn{1}{l}{} & & & \\
\hline Late complications & Correct ( $\mathrm{f}$ ) & Correct \% & Incorrect (f) & Incorrect \% \\
\hline Acute myocardial infarction & 58 & 97 & 2 & 3 \\
Hypertensive retinopathy & 51 & 85 & 9 & 15 \\
Paralysis & 54 & 90 & 6 & 10 \\
Physical deformity & 39 & 65 & 21 & 35 \\
& & & & \\
\hline
\end{tabular}

From table 5 Concerning immediate complication of uncontrolled hypertension respondents have poor knowledge as they provided nearly $50 \%$ of responses. However, nurses have moderate knowledge just above $50 \%$ regarding late complications of uncontrolled hypertension.

\section{CONCLUSION}

Hypertension is the leading cause of morbidity and mortality among the adult humans; no nation in the globe is immune to its fatal consequences. In Bangladesh, it is one of the 10 leading reported causes of death. Incidence and prevalence rate of hypertension are increased day by day. So, appropriate management techniques, preventive and control measures are needed. Nurses are playing vital role as a care provider, health educator and adviser for primitive, preventive and curative aspects of the patients. In this reason, as a care provider nurses need update knowledge on management of hypertension as well as prevention and control. Therefore, in order to address the national issues, the present study attempted to assess knowledge about management of hypertension.

From the demographic information, it has found that, majority of respondents were from elder age group. Largest proportion of respondents had passed secondary school level, however significant number of them had professional degree diploma in nursing. Very few numbers have higher education like MPH. Regarding the concept of hypertension, majority of respondents were able to de ne hypertension. However, significant proportion them were unable to identify factors and influential medicine that cause hypertension. Moreover, highest number of respondents was incapable in detecting all possible types of symptoms. Concerning the management and prevention of hypertension, respondents have comprehensive knowledge. On the other hand, respondents have moderate knowledge on complications of hypertension. According to the study, it can be said that, nurses have adequate knowledge concerning definition, management and prevention of hypertension. However, proper knowledge on causes and symptoms are required in order to management of patients. From this study, it could conclude that, authority might initiate opportunities for updating the nurses' knowledge on these areas through arranging the seminar/symposium/workshop/orientation programs etc.

\section{REFERENCES}

I. Juliet Addo, Liam Smeeth, and David A Leon. Hypertension in sub-saharan africa a systematic review. Hypertension, 50(6):1012-1018, 2007.

II. Jamy D Ard and Laura P Svetkey. Diet and blood pressure: Applying the evidence to clinical practice. American heart journal, 149(5):804-812, 2005. 
III. Kuldeep Kaur, Suresh K Sharma, Harpreet Jhajj, Gagandeep Kaur, and Harpreet Bajwa. Knowledge and learning needs of hypertensive patients visiting opd at dmc \& h, ludhiana. Nursing and Midwifery Research, 3(1), 2007.

IV. Bridgett Rahim-Williams, Joseph L Riley, Ameenah KK Williams, and Roger B Fillingim. A quantitative review of ethnic group differences in experimental pain response: Do biology, psychology, and culture matter? Pain Medicine, 13(4):522-540, 2012.

V. Md Shakhawat Hossain and Anna Martensson. Potential use of rhizobium spp. to improve fitness of nonnitrogen-fixing plants. Acta Agriculturae Scandinavica Section B-Soil and Plant Science, 58(4):352-358, 2008.

VI. Anthony J Viera, Lauren W Cohen, C Madeline Mitchell, and Philip D Sloane. High blood pressure knowledge among primary care patients with known hypertension: a north carolina family medicine research network (nc-fmrn) study. The Journal of the American Board of Family Medicine, 21(4):300-308, 2008.

VII. Unicef Who. Unfpa, the world bank. trends in maternal mortality: 1990 to 2010. World Health Organization, UNICEF, UNFPA, and The World Bank, 2012. 